\title{
Many reasons to talk about suicide
}

\section{Muitas Razões PaRa falarmos SOBRE SUICÍDIO}

\author{
Guilherme V. Polanczyk ${ }^{1 *}$ \\ Hospital das Clínicas, USP, São Paulo, SP, Brazil \\ *Correspondence: \\ Instituto de Psiquiatria, Hospital das Clínicas \\ Address: Rua Dr. Ovídio Pires de Campos, 785 \\ São Paulo, SP - Brazil \\ Postal code: $05403-010$ \\ gvp@usp.br
}

${ }^{1}$ Associate Professor, Department of Psychiatry, Faculdade de Medicina da Universidade de São Paulo (FMUSP). Head of the Inpatient Unit, Child and Adolescent Psychiatry Division, Institute of Psychiatry,

Approximately 1 million people die each year from suicide worldwide. ${ }^{1}$ This is the second leading cause of death among adolescents and young adults. ${ }^{1}$ Although recognized by the World Health Organization as a public health priority, mental disorders and suicide are beset by ignorance and stigma, which hinders proper treatment and prevention.

Recently, teenage suicide has been the subject of news because of a supposed online challenge called "Blue Whale," probably originating in Russia, which would encourage risky behavior among participants and ultimately suicide. It has also been widely discussed after being portrayed in an American drama series, 13 Reasons Why, which has been watched by many young people in Brazil and the world. Both the online challenge and the television drama pose risks, but the community's interest in the subject is an important opportunity to educate people about suicide.

The online challenge, in line with many websites, blogs and messaging lists that encourage other risky behaviors (such as self-mutilation and restrictive eating behavior) found its way in the lives of fragile adolescents who often already suffer from mental illnesses. Associated with psychopathology, these adolescents frequently face social isolation and are part of families that are unable to identify and deal with their difficulties. While using digital tools, adolescents somehow feel understood and belonging to a social group. Dysfunctional symptoms and behaviors are thus justified and encouraged, and ultimately intensified.

The drama series, on the other hand, has a less explicit potential to stimulate suicidal behavior, even though its producers advocate a positive effect on young people. The plot portrays suicide as a glamorized act that is the consequence of specific events and cannot be prevented, achieving specific functions successfully, which is mainly revenge. Also, suicide is shown explicitly, emotionally impacting any viewer, especially the most fragile ones. In view of these characteristics, ${ }^{2}$ the experience of a fiction narrative such as these for those who already have risk factors for suicide, including depression, family history of suicide, previous suicide attempts or previous self-injury, suicidal ideation and lack of social support, can actually represent encouragement for suicidal behavior, especially in the presence of other triggers, such as access to lethal methods, impulsivity, substance abuse and acute stress events. ${ }^{3}$

Suicide is still marked by ignorance and stigma not only in society as a whole, but also within the medical community, both in relation to the suicide of patients and the physicians themselves. It is estimated that $45 \%$ of people who commit suicide consulted a physician in the month prior to their death, and there is rarely any documentation of suicide risk assessment. ${ }^{4}$ Many doctors mistakenly think that those who talk about suicide do not really want to kill themselves, that asking about suicidal intent and plan for those who feel depressed may encourage suicide, or that people do not want to talk about their thoughts about death. These are all myths that interfere with proper evaluation and management of the cases.

As for physician suicide, male doctors have a 1.41 times higher rate than the general male population, while female doctors have a 2.27 times higher rate than that observed in the general female population. ${ }^{5}$ Surprisingly, despite substantially higher rates of depression ${ }^{6}$ compared to the general population, and also suicide, ${ }^{5}$ depressed students and physicians ${ }^{6}$ and suicide victims ${ }^{7}$ have lower treatment rates. Among the reasons for not seeking treatment, stigma and self-stigma, denial of the presence of depression and fear of the negative impact that psychiatric treatment may have on performance and professional image in an extremely competitive environment have a strong effect. In addition to depression, suicide risk factors among medical students 
and physicians include temperament characteristics such as perfectionism, being too demanding of themselves, and rigid cognitive models such as not allowing error and not placing oneself in the position of those who need care. In addition, pressure at work, conflict between family and patient dedication and career, burnout, and sleep restriction are important risk factors. ${ }^{8,9}$

In order to reduce suicide rates globally, it is necessary to reduce ignorance and stigma about mental disorders. Recently, the World Health Organization has taken an important step in this direction. For the first time, the WHO chose, as a theme for World Health Day (04/07/2017), a mental health condition: depression. The campaign slogan, "Let's Talk," emphasizes the importance of reducing stigma and depression. Medical doctors, regardless of specialty, should be aware of the mental health of their patients - as well as their own - and suicide risk should be evaluated whenever indicated. Anti-suicide strategies need to be part of public health policies, as well as school and university policies. A change in medical culture - regarding the requirements of training, the balance between professional and personal life, and the ways that the profession can affect a doctor's mental health - is in order..$^{10}$ Doctors seeking and offering help for mental suffering should be culturally accepted and encouraged. Silence, shame and fear are great obstacles to psychiatric care that need to be removed.

\section{References}

1. WHO. Mental health: suicide prevention. 2014 [cited 2017 May 31]. Available from: http://www.who.int/mental_health/suicide-prevention/en/.

2. Gould G, Jamieson P, Romer D. Media contagion and suicide among the young. Am Behav Scient. 2003; 46(9):1269-84.

3. Hawton K, Saunders K, O'Connor R. Self-harm and suicide in adolescents. Lancet. 2012; 379(9834):2373-82.

4. Ahmedani BK, Simon GE, Stewart C, Beck A, Waitzfelder BE, et al. Health care contacts in the year before suicide death. J Gen Intern Med. 2014; 29(6):870-7.

5. Schernhammer E, Colditz G. Suicide rates among physicians: a quantitative and gender assessment (meta-analysis). Am J Psychiatry. 2004; 161(12):2295-302.

6. Mata DA, Ramos MA, Bansal N, Khan R, Guille C, Di Angelantonio E, et al. Prevalence of depression and depressive symptoms among resident physicians: a systematic review and meta-analysis. JAMA. 2015; 314(22):2373-83.

7. Gold KJ, Sen A, Schwenk TL. Details on suicide among U.S. physicians: data from the National Violent Death Reporting System. Gen Hosp Psychiatry. 2013; 35(1):45-9.

8. American Foundation for Suicide Prevention. Physician and medical student depression and suicide prevention [cited 2017 May 31]. Available from: https://afsp.org/our-work/education/physician-medical-student-depressionsuicide-prevention.

9. National Academy of Sciences. Breaking the culture of silence on physician suicide. 2016.

10. Muller D. Kathryn. N Engl J Med. 2017; 376(12):1101-1103. doi: 10.1056/ NEJMp1615141. 